\section{Die Möglichkeiten des ökologischen Marketings in der nördlichen Region der ungarischen Tiefebene ${ }^{1}$}

\author{
Maria Szolnoki Karkus \\ Universität Debrecen, \\ Lehrstuhl für Agrarökonomie und Volkswirtschaftslehre, \\ Debrecen \\ szolnokine@helios.date.hu
}

„Die ökologischen Probleme von heute werden zu ökologischen Wettbewerbsfeldern von morgen. “

(Dyllick)

\section{ZUSAMMENFASSUNG}

Diese Studie sucht die Antwort auf die Frage, welche Chancen das ökologische Marketing in der Nördlichen Region der Tiefebene in Ungarn haben kann. Wir haben in den Jahren 1996 und 2003 in unserer Region eine empirische Untersuchung anhand eines standardisierten Fragebogens, und danach persönliche Interviews im Kreis der Unternehmensleiter der Lebensmittelbranche durchgeführt. Aus beiden Untersuchungen hat es sich herausgestellt, dass der Umweltschutz nur durch Gesetzesvorschriften den Unternehmen aufgezwungen wird. Die Anregung des Marktes und der Medien hat eine geringere Bedeutung. Der Umweltschutz liegt unter den Zielen des Unternehmens auf dem letzten Platz. Die Unternehmensleiter meinen: die Stärkung der Rolle des Umweltschutzes würde die Kosten des Unternehmens steigern und kurzfristig den Profit in geringem Maße vermindern.

Schlüsselworte: Ökomarketing, Lebensmittelbranche, Ökologische Belastungen, Ökologische Ansprüche, Ökologische Wettbewerbsfelder, Umweltorientierte Absatzmassnahmen, Wettbewerbsposition

\section{EINLEITUNG}

Die Vermehrung der globalen Umweltprobleme und der lokalen Umwelteinflüsse stellen für die Wissenschaft, die Forschung, aber auch für die Teilnehmer des wirtschaftlichen Lebens eine ernste Herausforderung dar. Als eine mögliche Antwort der Unternehmen auf diese Herausforderung kann die Ökologisierung ihres Marketings sein.

Die in den einzelnen Wirtschaftszweigen von den Unternehmen (durch ihre Tätigkeit, ihre Produkte) verursachten und erlittenen Umwelteinflüsse drücken zusammen die Betroffenheit der Wirtschaftszweige durch die Umweltproblematik aus. Die Nahrungsmittelwirtschaft und darin die Landwirtschaft und die Nahrungsmittelindustrie sind davon bedeutend und in dem letzten Jahrzehnt sowohl in Europa als auch in Ungarn zunehmend berührt (Meffert und Kirchgeorg, 1998).

\footnotetext{
1 Unsere Studie wurde mit der Unterstützung der wissenschaftlichen Organisation OTKA (T 031990) zusammengestellt
}

Ungarn und die landwirtschaftlichen Betriebe der den Gegenstand unserer Untersuchung bildenden nördlichen Region der ungarischen Tiefebene sollen diese Tatsache vor dem Beitritt Ungarns an die Europäische Union berücksichtigen und sich auf die Herausforderung vorbereiten.

Die nördliche Region der Tiefebene umfasst den nordöstlichen Teil Ungarns, etwa $19,1 \%$ des Territoriums mit 15,3\% der Bevölkerung des Landes. Die Region setzt sich aus drei Verwaltungsbezirken (Hajdú-Bihar, Jász-Nagykun-Szolnok und SzabolcsSzatmár-Bereg) zusammen. Sie ist ein Flachland mit guten Gegebenheiten für die landwirtschaftliche und die Nahrungsmittelproduktion.

Die Fakultät für Agrarökonomie und Entwicklung ländlicher Räume an der Universität Debrecen, wo die Verfasserin dieser Abhandlung ihre pädagogische und Forschungsarbeit verrichtet, ist das geistige und wissenschaftliche Zentrum dieser Region.

Diese Studie sucht die Antwort auf die Frage, welche Chancen das ökologische Marketing in dieser Region haben kann. Da eine begründete Antwort auf diese Frage nur von den Unternehmensleitern erwartet werden kann, haben wir die Leiter des Nahrungsmittelsektors gefragt, wie sie die Beziehungen zwischen Wirtschaft und Umweltschutz beurteilen. Wir haben dazu einen Fragebogen zusammengestellt, aus dem wir die wichtigsten Charakterzüge des Unternehmens sowie die Meinung der Unternehmensleiter, ihre subjektiven Schätzungen über die wirtschaftlichen, finanziellen Konsequenzen der umweltorientierten Unternehmensleitung und des Marketingmanagements kennenlernen können. Wir versuchten auch die günstigen und ungünstigen Wirkungen zahlenmäßig zu bestimmen.

\section{FORSCHUNGSZIEL UND FORSCHUNGS- KONZEPT}

Die Unternehmensführung widmet dem Umweltschutz große Aufmerksamkeit, baut ihn in ihre strategischen Ziele nur dann ein und führt ihre gesamte wirtschaftliche Tätigkeit unter Einbeziehung der Gesichtspunkte des Umweltschutzes nur dann aus, wenn sie dazu gezwungen ist oder wenn sie davon Marktvorteile, Wettbewerbsvorteile, Profit erhofft.

Die Unternehmensleitung wird über ihre eigene „Umweltbetroffenheit“ durch verschiedene Repräsentanten der Wirtschaft und der Politik in Kenntnis gesetzt, die den sogenannten 
„Umweltinteressen“"

folgen.

interessendurchsetzende Fähigkeit

Die

dieser wirtschaftlichen, gesellschaftlichen, politischen Organisationen, die stimulierende Kraft kann in den einzelnen Wirtschaftszweigen sehr unterschiedlich sein (Dyllick und Belz, 1996).

Aufgrund der gedankenerweckenden Publikationen von Meffert und Ostmeier (1990), Dyllick (1989) sowie von Belz (2001) haben wir in den Jahren 1996 und Anfang 2003 in unserer Region eine empirische Untersuchung im Kreis der Unternehmensleiter der Nahrungsmittelindustrie und der Landwirtschaft durchgeführt. Wir wollten ihre Meinung anhand persönlicher Interviews und eines standardisierten Fragebogens darüber kennenlernen, vor welche Herausforderungen von der Umwelt sie persönlich, ihre Tätigkeit und ihre Produkte gestellt werden, woher diese Herausforderungen kommen und wie stark sie sind.

Wir haben uns auch danach erkundigt, ob sie den Umweltschutz in ihrer Firma als ein gewichtiges strategisches Ziel ansehen, ob sie die Stärkung dessen Rolle für wichtig halten; wenn ja, warum und wenn nicht, was sie daran hindert. Wir wollten erfahren, wie die Unternehmensleiter die Auswirkungen der Stärkung des ökologischen Marketings auf die wirtschaftliche Tätigkeit ihres Betriebes beurteilen.

Da wir uns das Ziel gesetzt haben, neben den gewöhnlichen alternativen Antworten möglicherweise auch quantifizierbare Ergebnisse zu bekommen, mussten die Befragten die Stärke der Wirkung mit Zahlen bewerten (im allgemeinen zwischen 0 und +3 , und wenn auch die negative Wirkung interpretierbar ist, zwischen 3 und +3 ). So konnten wir bei der Verarbeitung der Fragebögen nicht nur das Vorhandensein der Wirkung und deren positive oder negative Richtung feststellen, sondern auch „die Stärke“ des Zusammenhanges bewerten.

Während die Anzeichen für die Verbreitung der ökologischen Anschauung in der Fachliteratur des ungarischen Marketings und der Unternehmensführung bereits bemerkbar sind (Zsolnai, 1994; Kósi, 1996; Kovács, 1999; Németh, 1999; Csutora, 2001; Kálmán, 2002), wird davon in der Praxis der Unternehmen noch sehr wenig verwirklicht. Wir haben dieselbe Erfahrung gemacht und sie wurde durch die Ergebnisse unserer ersten Umfrage bestätigt. Wir haben auf das Ergebnis sehr neugierig gewartet, in welcher Richtung sich die „Situation“"geändert hat.

\section{PLANUNG UND AUFBAU DER EMPIRISCHEN UNTERSUCHUNGEN}

Die erste Untersuchung wurde im Herbst 1996 durchgeführt. Wir haben unsere Fragebögen im Bezirk Hajdú-Bihar an zwölf Unternehmensleiter gesandt und davon sieben wieder bekommen. Wir haben unsere Untersuchung im Frühjahr 2003 praktisch in unveränderter Form wiederholt und auf die Region der nördlichen Tiefebene ausgebreitet. Von den 30 Fragebögen haben wir 15 wieder bekommen. Wir haben allen Unternehmen einen Fragebogen geschickt, die wir in unsere erste Untersuchung einbezogen haben. Von diesen letzteren sieben Firmen haben jetzt vier geantwortet. Die untersuchte Menge hat sich also verändert. Gleichzeitig macht die höhere Zahl der antwortenden Unternehmensleiter die als „Anzeichen“ interpretierten Ergebnisse der früheren Untersuchung glaubhafter.

Wir haben die Ergebnisse unserer gegenwärtigen Untersuchung auch an und für sich selbst als bemerkenswert gefunden, doch haben wir diese nach Möglichkeit mit den Ergebnissen unserer früheren, in einem engeren Kreis durchgeführten Untersuchung sowie fallweise mit den Ergebnissen der Untersuchungen in Deutschland verglichen (Meffert und Ostmeier, 1990) und versuchten die Ähnlichkeiten und die Unterschiede sowie die in der Zwischenzeit abgelaufenen Veränderungen aufzuschließen.

Wir haben uns mit der Untersuchung zum Ziel gesetzt, ein genaueres und glaubhafteres Bild über die Chancen des ökologischen Marketings in unserer Region, in dem nördlichen Teil der Tiefebene über die landwirtschaftlichen und lebensmittelverarbeitenden Betriebe zu erhalten. Der Gang der Forschung ist in Abbildung 1 veranschaulicht. Wir haben die Fragen auf solche Weise zusammengestellt, dass wir die Ausgangssituation, die wichtigeren Charakterzüge der Betriebe sowie die Meinungen und Schätzungen der Firmenleiter darüber kennenlernen können, wie sie die wirtschaftlichen, finanziellen Konsequenzen der umweltorientierten Unternehmensführung und des Marketingmanagements beurteilen.

Die 35 Fragen bilden vier Blöcke. Die ersten 11 Fragen des Fragebogens (A) beziehen sich auf die wirtschaftliche Situation des Unternehmens, auf die Umweltprobleme. Die nächsten 10 Fragen (B) wollen ausfindig machen, wie der den Fragebogen ausfüllende Firmenleiter den Zustand des Umweltschutzes, der Umweltpolitik in seiner engeren Umgebung, beziehungsweise allgemein in Ungarn einschätzt.

Wir haben den Unternehmensleiter bei der Beantwortung der sechs Fragen des dritten Blocks um eine zahlenmäßige Schätzung gebeten, wie er den Umweltschutz in seinem Sektor, beziehungsweise in seiner eigenen Firma bewertet.

Schließlich (D) haben wir den Unternehmensleiter gebeten $\mathrm{zu}$ beurteilen, welche Auswirkung es zur Folge auf die wirtschaftliche Leistung des Unternehmens hätte (Umsatz, Gewinn, Marktbeteiligung, Wachstumschancen, Wettbewerbsfähigkeit), wenn das Unternehmen auf den Umweltschutz in den Werbungen, in dem Umsatz, in der Produkt- und Preispolitik einen stärkeren Akzent setzen würde. 
Abbildung 1: Struktur des Fragebogens, Gang der Untersuchung

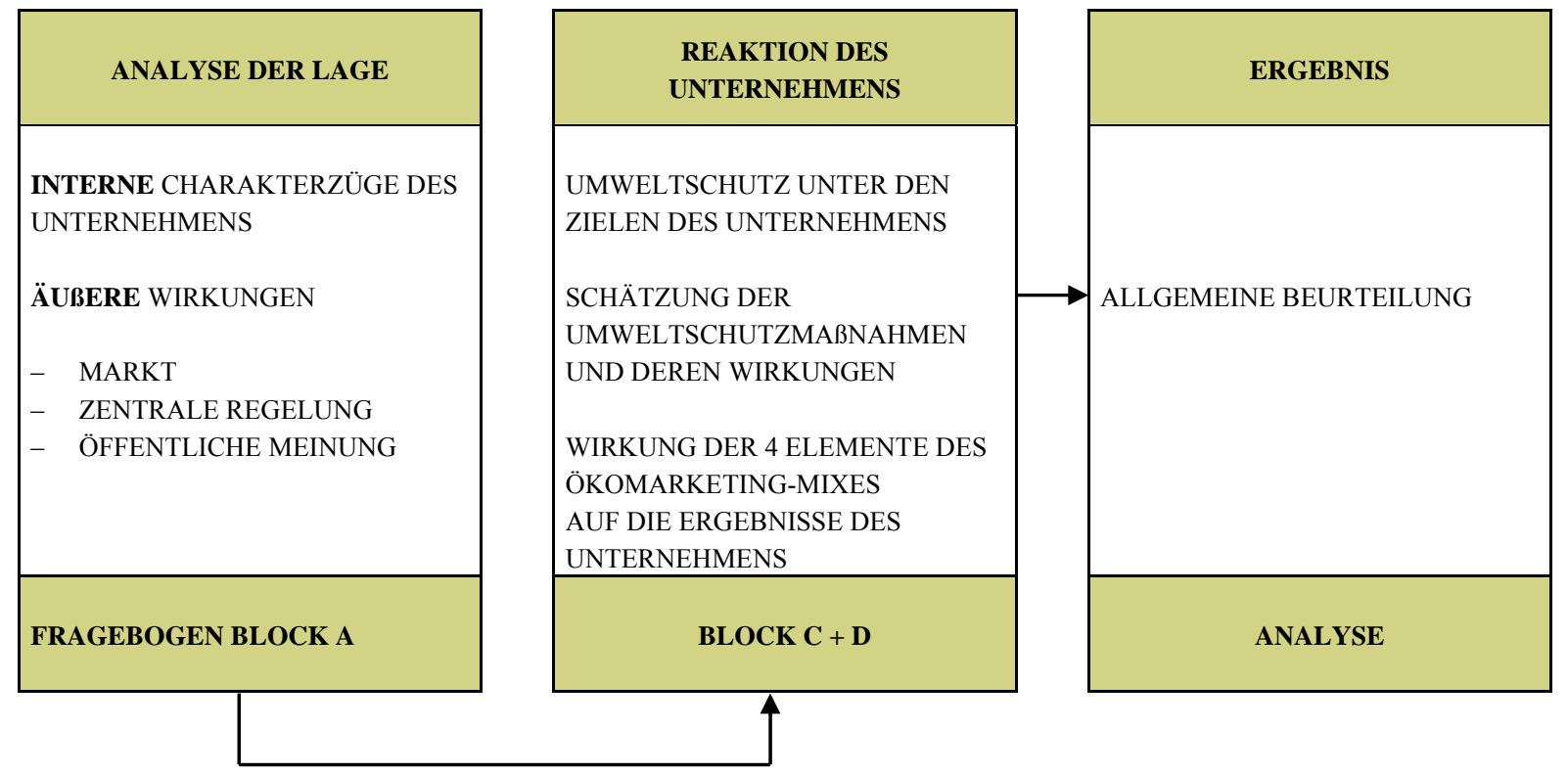

\section{BEWERTUNG DER INFORMATIONEN DER FRAGEBÖGEN}

\section{Wichtigere Charakterzüge der Unternehmen (A)}

Die Informationen liefernden Unternehmen haben bei den beiden Untersuchungen auf Grund ihres Alters, ihrer Größe, ihres Tätigkeitsbereiches ein abweichendes Bild aufgewiesen.

Das Bild kann auch in der Hinsicht der Gesellschaftsform der Unternehmen als bunt bezeichnet werden: während bei der ersten Untersuchung 3 Aktiengesellschaften, 3 Gesellschaften mit beschränkter Haftung und eine Genossenschaft ihre Meinung äußerten, konnten wir jetzt die Meinung von 8 Aktiengesellschaften, 5 Gesellschaften $\mathrm{mbH}$, einer Gemeinnützigen Gesellschaft und einer Genossenschaft neuen Typs kennenlernen.

Die Größe der Unternehmen weist ebenfalls eine starke Streuung auf: in ihrem eigenen Sektor haben sich im Jahr 19963 Unternehmen für mittelgroß, eines für klein gehalten. Im Jahr 2003 haben sich 3 Unternehmen als groß, 9 als mittelgroß, eines als klein klassifiziert. Zwei Unternehmensleiter haben diese Frage nicht beantwortet.

Nach den Tätigkeitsbereichen sieht das Bild folgendermaßen aus: Fleisch- und Salamierzeugung als primäre und sekundäre Tätigkeit, Erzeugung und Absatz von Backwaren und Konditorwaren, Herstellung und Handel von Milchprodukten, Kühlung und Lagerung von Obst- und Gemüseprodukten. Dieses abwechslungsreiche Bild hat sich jetzt mit der Erzeugung und Abfüllung von alkoholischen und Erfrischungsgetränken, ferner mit Tätigkeiten in der Mühlenindustrie und in der Landwirtschaft erweitert.

Die vorkommenden und behandlungsbedürftigen Umweltprobleme sind entsprechend dem
Tätigkeitsbereich vielfältig gewesen und geblieben. Die größten Sorgen sind in der Abfallwirtschaft und der Wasserwirtschaft in erster Linie in den Erzeugungsprozessen aufgetaucht und dies stimmt mit den Feststellungen der Fachliteratur überein (Belz, 2001). In dem eigenen Unternehmen wird auf die Fragen der Abfallwirtschaft, auf die mit dem Rohstoff- und Energieverbrauch und der Verpackung zusammenhängenden Umweltprobleme ein stärkerer Akzent gesetzt.

\section{Beurteilung der Lage des Umweltschutzes (B)}

Die Meinung der Unternehmensleiter über die Frage, ob man sich in Ungarn mit dem Umweltschutz zu viel oder zu wenig beschäftigt, ist auch jetzt wie früher unterschiedlich. Ebenso unterschiedlich äußern sie sich über die Wirksamkeit der strengen Rechtsvorschriften und über die einseitig emotionelle Einstellung der Umweltschutzbewegungen.

In der Frage, dass die Technik die Umweltprobleme von sich selbst auch in Zukunft nicht lösen wird, herrscht Einverständnis. Jedoch besteht hier ein Unterschied: während die entscheidende Mehrzahl früher bezweifelt hat, dass der Zustand der Umwelt bei uns besser wird, waren die Befragten jetzt viel optimistischer. Gleichzeitig halten alle einstimmig die Stärkung der Erziehung in den Schulen zum umweltbewussten Verhalten für wichtig. Während die Befragten früher einstimmig meinten, dass die Bürger bei uns nicht geneigt sind, größere Opfer als sie heute sind für eine bessere Umwelt zu bringen, erklärten jetzt beinahe $50 \%$ der Unternehmensleiter, dass sie bereit sind, Opfer für eine schönere Umwelt zu bringen.

Wir haben eine bemerkenswerte Änderung gefunden: während die Mehrheit früher meinte, dass die internationale Wettbewerbsfähigkeit durch die Verschärfung der Anforderungen der Umweltpolitik 
nicht gefährdet wird, hat jetzt die entscheidende Mehrheit auf diese Frage mit „Ja“ geantwortet.

Die Unternehmen unterliegen verschiedenen Wirkungen, durch die sie in unterschiedlichem Maß zur Ausgestaltung der umweltorientierten Firmenführung angeregt werden. Aufgrund der Antworten kann in Ungarn für die Nahrungsmittelindustrie folgendes festgestellt werden (Abbildung 2):

- die Unternehmen werden zur umweltorientierten Führung in erster Linie kraft Gesetzes angeregt $(2,45)$,

- als zweiter Wirkungsfaktor in der Rangordnung können die Erwartungen der ausländischen Kunden in Betracht gezogen werden,

- die Unternehmen lassen sich durch die Tätigkeit der Konkurrenten, die Kritik der Medien nur mittelmäßig beeinflussen,

- die Ansprüche der heimischen Kunden und die Aktivitäten der Umweltschutzbewegungen regen etwas weniger zum umweltorientierten Verhalten an.

Wir teilen in Abbildung 2 die Ergebnisse unserer beiden Untersuchungen sowie die vergleichbaren, korrigierten Angaben der von Meffert und Ostmeier in Deutschland, im Jahre 1990 durchgeführten Untersuchungen mit. Die Abbildung zeugt von einer positiven Änderung in den vergangenen 7 Jahren. Die Reihenfolge der Wirkungsfaktoren ist zwar unverändert geblieben, jedoch ist die stimulierende Wirkung stärker geworden.

Abbildung 2: Zum umweltorientierten Verhalten anregende Faktoren und ihre Wirkung in Ungarn (in den Jahren 1996 und 2006 ) und in Deutschland (im Jahr 1988)

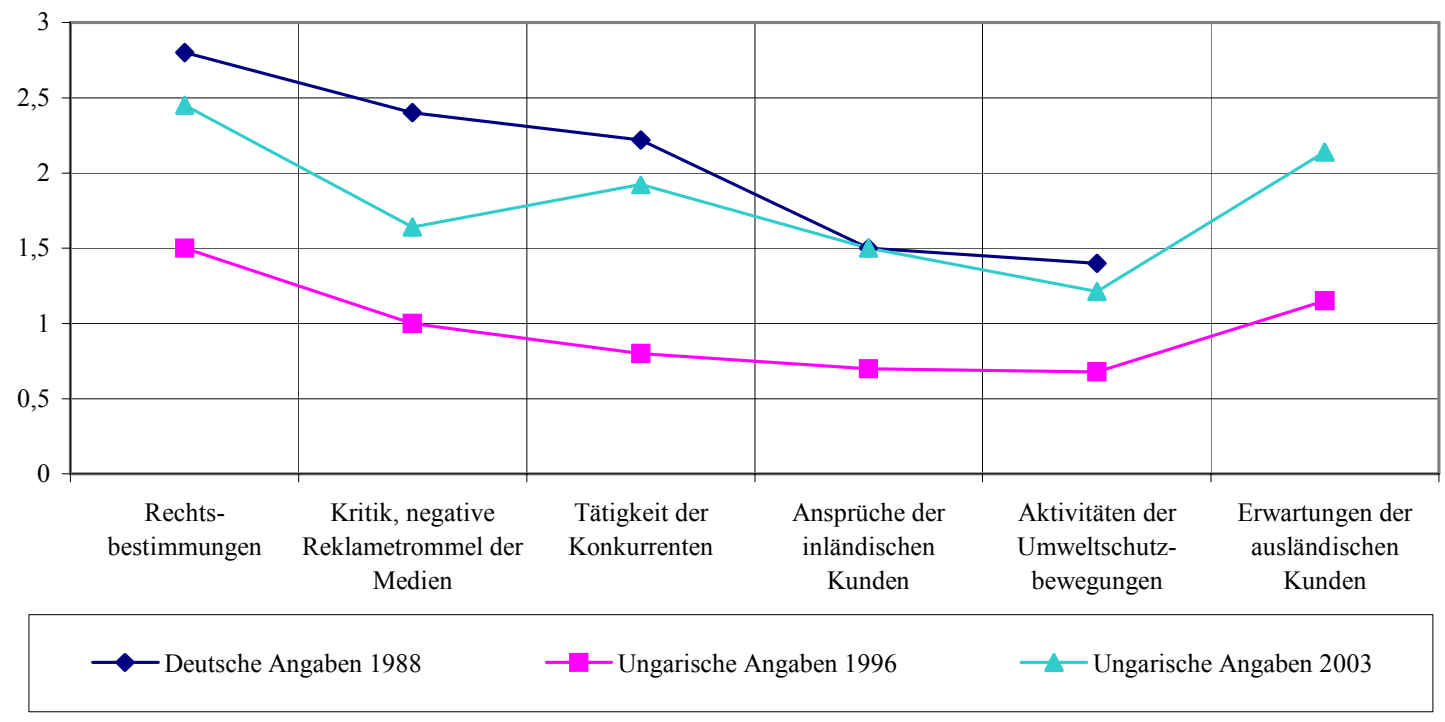

Der Platz des Umweltschutzes unter den Zielen des Unternehmens $(C)$

Die nächste Gruppe der Fragen verlangte von den Unternehmensleitern eine individuelle Bewertung, eine subjektive Schätzung. Als erstes wollten wir feststellen, wohin der Umweltschutz unter den Zielen der Firma platziert wird. Die Wichtigkeit der Ziele konnte mit Punkten von 0-3 benotet werden (der höhere Wert zeugt von größerer Bedeutung). Als zweites haben wir um Antwort auf die Frage gebeten, wie es die Ziele des Unternehmens beeinflussen würde, wenn die Firma auf den Umweltschutz einen größeren Wert läge, wie es heute der Fall ist. Wir stellen die Antworten auf die beiden Fragen in den Abbildungen 3 und 4 vor.

In Abbildung 3 ist es ersichtlich, dass sich in der Beurteilung der Unternehmensziele gegenwärtig kein signifikanter Unterschied zeigt. Die kurzfristige Steigerung des Profits, die Erhaltung der Arbeitsplätze und der Erwerb neuer Märkte haben vielleicht einen um eine Nuance niedrigeren Punktwert bekommen (2,53 beziehungsweise 2,61). Der Punktwert des Umweltschutzes $(2,69)$ ist hinter dem höchstbewerteten Ziel, der Steigerung der Produktion $(2,84)$ nur geringfügig zurückgeblieben. Eine wesentliche Änderung hat sich im Vergleich zu der Untersuchung im Jahr 1996 vollzogen und eine den Ergebnissen der deutschen Untersuchung ähnliche Teilung ist in der Hinsicht der Unternehmensziele erschienen.

Die Ursache für die positive Änderung kann nach unserer Ansicht in der Konsolidierung der Marktwirtschaft, in der Verbreitung und Verschärfung der Umweltschutzbestimmungen, in der Entwicklung des Umweltbewusstseins und darin gesucht werden, dass die Unternehmensleiter erkannt haben, was die Gesellschaft von ihnen erwartet.

Die günstigste Wirkung würde sie aber auf den Ruf des Unternehmens ausüben $(1,15)$ und sie würde gewissermaßen auch die Erhaltung der Arbeitsplätze begünstigen. Wir illustrieren die Ergebnisse der beiden Untersuchungen in Abbildung 4. Da die Unternehmensleiter das Ausmaß der Wirkung mit Punkten von 3 bis +3 bewerten konnten, sind in das Diagramm durchschnittliche Punktwerte eingestellt worden. 
Die Umweltschutzziele sind in die strategischen Pläne der Unternehmen eingebaut, sie stehen bei zwei Unternehmen unter den wichtigsten Zielen, aber sie erscheinen - zwar anderen Zielen untergeordnet auch bei den anderen. Eine umweltschutzbezogene Durchleuchtung wurde lediglich bei einer einzigen
Firma vorgenommen: der ausländische Käufer hat dies vor dem Kauf der Firma für wichtig gehalten. Aufgrund der Durchleuchtung wurden in einigen Bereichen Empfehlungen zur Verbesserung des Umweltschutzes erteilt.

Abbildung 3: Bewertung der Unternehmensziele (zwischen 0 und 3) in Ungarn (1996; 2003) und in Deutschland (1988)

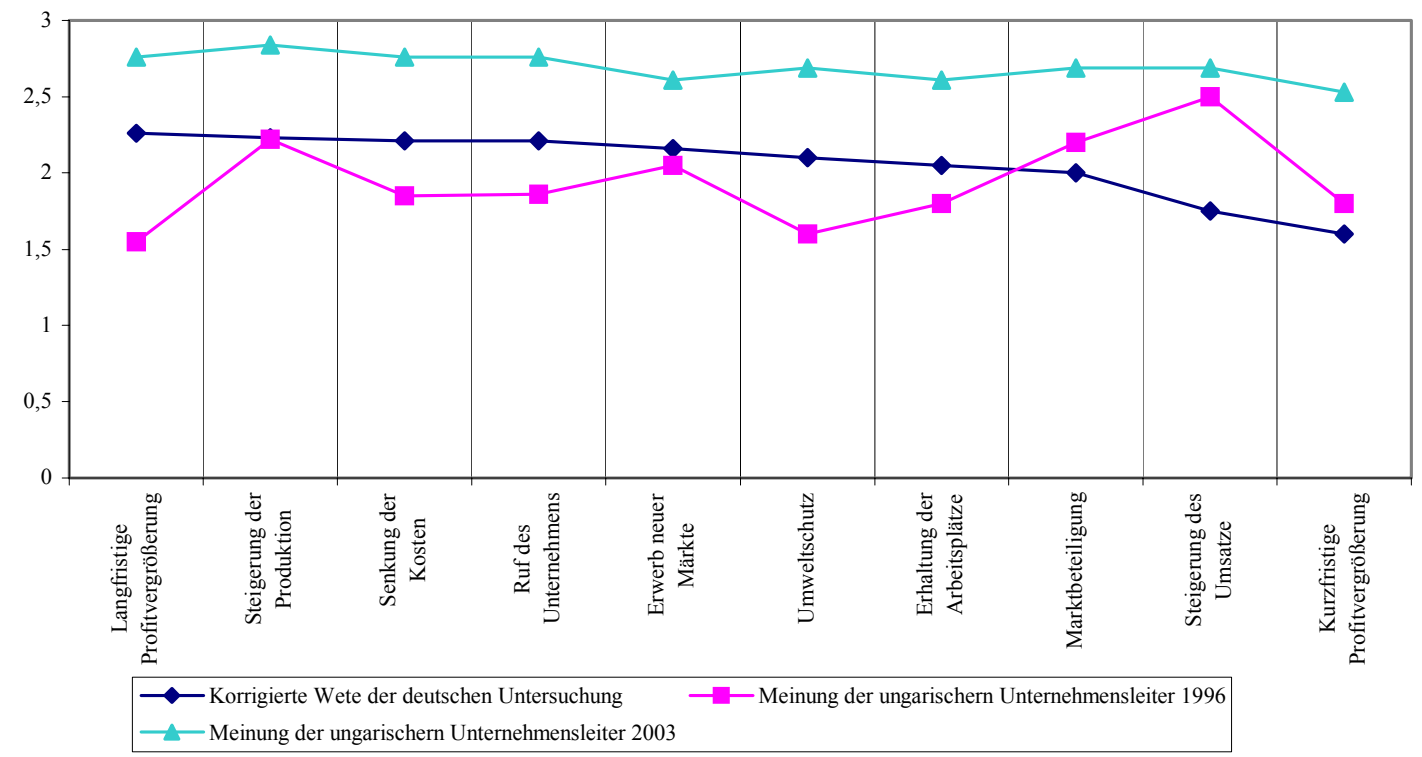

Abbildung 4: Geschätzte durchschnittliche Wirkung des Umweltschutzes auf die einzelnen Ziele des Unternehmens in den Jahren 1996 und 2003

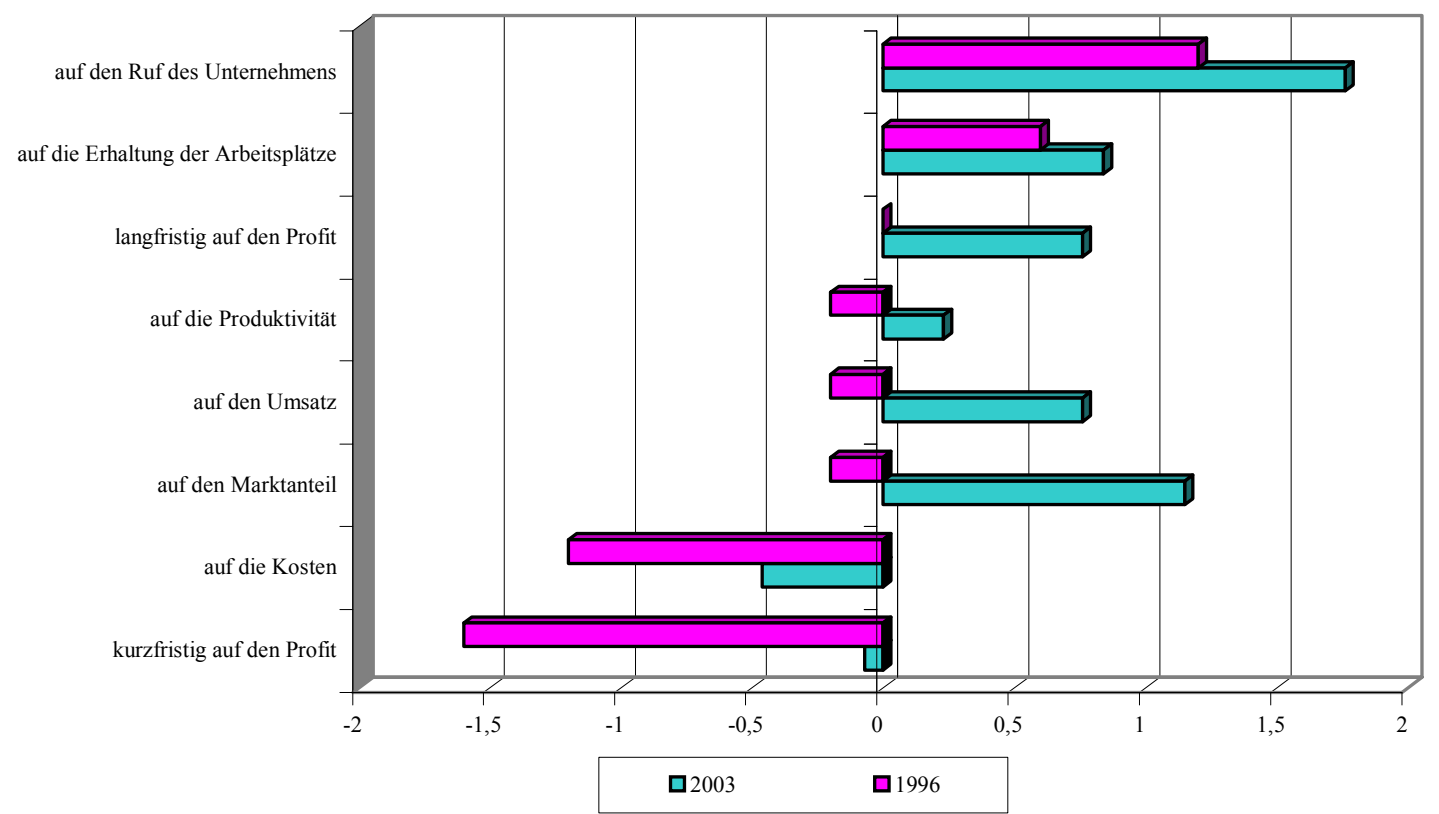

Umweltschutz in den einzelnen Elementen des Marketing-mixes (D)

Wir haben einige komplizierte Fragen gestellt, deren Beantwortung den Befragten vor eine schwere Aufgabe gestellt hat. Wir baten ihn mit Punkten zwischen 3 und +3 zu bewerten, welchen Einfluss die Tatsache auf die Ziele des Unternehmens nähme, wenn sich die umweltorientierte Betrachtungsweise bei den 4 Elementen des Marketing-mixes verstärken würde. Wir bewerten nachstehend die Ergebnisse der beiden Untersuchungen zusammen.

Als erstes wollten wir erfahren, wie es den Umsatz, den Gewinn, die Marktbeteiligung, die Wettbewerbsfähigkeit des Unternehmens 
beeinflussen würde, wenn die umweltfreundlichen Eigenschaften des Produktes, die umweltschonende Leistung und Verpflichtung in der Werbung stärker betont würden. Die Stärke der Wirkung wurde früher mit Punkten zwischen $+0,5$ und $+0,7$, jetzt aber mit $+0,85$ und $+1,42$ benotet. Die Reihenfolge hat sich nicht verändert: diese Maßnahme würde am besten den Umsatz, die Marktbeteiligung und die Wachstumschancen des Unternehmens verbessern.

Als zweites wollten wir wissen, welche Auswirkungen in den Ergebnissen des Unternehmens erwartet werden könnten, wenn die Gesichtspunkte des Umweltschutzes in dem Vertrieb und dem Transport stärker als bisher zur Geltung kämen. In der früheren Untersuchung wurde einstimmig gemeint, dass diese Tatsache den Gewinn des Unternehmens verringern $(0,2$ und $+0,5)$ und die Wettbewerbsfähigkeit in geringem $\mathrm{Ma}$ verbessern würde $(+0,7)$. Nach der zweiten Untersuchung wäre die Wirkung überall günstig, die Konkurrenzfähigkeit wurde mit +Eins, der Gewinn mit $+0,2$ bewertet.

Die dritte Frage richtete sich auf die Produktpolitik. Die Befragten haben die wirtschaftliche Wirkung der Entwicklung umweltschonender Produkte und Techniken, beziehungsweise die der umweltfreundlichen Verpackung sowohl in der ersten als auch in der zweiten Untersuchung als günstig beurteilt. Der geschätzte Wert der Wirkung ist jetzt mit einer einzigen Ausnahme höher und liegt zwischen +0,78 und $+1,21$ Punkten: die Wirkung auf das Wachstum der Konkurrenzfähigkeit und des Umsatzes wird an erster Stelle erwähnt und gleich danach folgen die Wachstumschancen.

Die Beurteilung der sich in der vierten Komponente des Marketing-mixes, in den Preisen widerspiegelnden Umweltwirkungen wies und weist ziemlich große Unterschiede auf (die Punktzahlen liegen zwischen 0,28 und $+0,42$ ). Die Meinungen sind nicht einmal in der Hinsicht der mathematischen Vorzeichen einheitlich.

\section{SCHLUSSFOLGERUNGEN}

1. Es hat sich aus der Untersuchung herausgestellt, dass der Umweltschutz in Abhängigkeit von Tätigkeitsbereich, Größe und Außenhandelsbeziehungen Sorgen bereitet. Die Unternehmensleiter nehmen die zunehmende Bedeutung des Umweltschutzes nachdrücklich wahr und drücken dies in ihren Meinungen aus. Während die Meinung früher, im Jahr 1996 einstimmig war, dass sich der Zustand der Umwelt in den nächsten Jahren in Ungarn nicht verbessern werde und die Unternehmensleiter bezweifelten die günstigen Wirkungen sowohl der Technik als auch der umweltpolitischen Regelung, haben sie sich jetzt darüber ausgesprochen optimistisch geäußert. Während sie die Verschärfung des Umweltschutzes früher für notwendig hielten - allerdings nicht für ihr Unternehmen - fordern dies jetzt lediglich 50\% der Unternehmensleiter. Während die Mehrheit früher der Meinung war, dass die Bürger nicht geneigt seien, größere Opfer für eine bessere Umwelt $\mathrm{zu}$ bringen, ist heute die Hälfte der Befragten der Ansicht, dass wir bereit sind, Opfer für die saubere Umwelt zu bringen.

2. Aus der früheren Untersuchung hat es sich herausgestellt, dass der Umweltschutz unter den Zielen des Unternehmens auf dem letzten Platz liegt. Jedoch widerspricht dieser Feststellung die Meinung, dass der Umweltschutz nach der Meinung der Mehrheit unter den wichtigsten Unternehmenszielen stehen sollte. Aus der gegenwärtigen Untersuchung kann die Schlussfolgerung gezogen werden, dass es in der Beurteilung der Unternehmensziele keine signifikante Abweichung gibt. Die Punktzahl des Umweltschutzes liegt kaum etwas hinter den der höchstbewerteten Ziele.

3. Die Unternehmensleiter meinen: die Stärkung der Rolle des Umweltschutzes würde die Kosten des Unternehmens steigern und kurzfristig den Profit in geringem Maße vermindern. Das Unternehmen würde sich einen besseren Ruf erwerben, könnte die Arbeitsplätze erhalten und seinen Marktanteil vergrößern. Wir haben im Vergleich zu unserer früheren Untersuchung eine bedeutende Veränderung erfahren: die günstigen Wirkungen haben sich verstärkt, die ungünstigen haben sich gemäßigt.

4. Die Unternehmensleiter begegnen den Wirkungen aus verschiedenen Richtungen her: einige hindern, andere fördern die Aktivitäten in dem Umweltschutz. In Ungarn, in der Nahrungsmittelindustrie wird der Umweltschutz durch Gesetzesvorschriften den Unternehmen aufgezwungen. Darüber hinaus wirken auch die Erwartungen der ausländischen Käufer und die Aktivitäten der Konkurrenten in dieser Richtung. Die Anregung des heimischen Marktes und der Medien hat eine geringere Bedeutung. Die Wichtigkeitsfolge der Faktoren ist unverändert geblieben, jedoch hat die Stärke der anregenden Wirkung beträchtlich zugenommen.

Die wichtigsten Konsequenzen aufgrund der mündlichen Interviews

Alle fünf befragten Personen sind Agrarfachleute und Unternehmer. Zwei von ihnen bekleiden einen führenden Posten in einer Interessenvertretung und bewirtschaften einen eigenen Betrieb (sie haben ihre Meinung in dieser Doppelrolle geäußert). Wir stellen von den kritischen Antworten unserer Gesprächspartner - wegen Umfangsbeschränkungen - lediglich einige, den Umweltschutz beschränkende Gesichtspunkte, konkrete Gedanken vor.

1. Unter den Bewusstseinfaktoren haben mehrere erwähnt: den Mangel an Kenntnissen, die falschen Routinen, das Fehlen des umweltorientierten Denkens, das Bagatellisieren der Umweltgefahren sowie dass die Bedeutung der Erfordernisse der Nahrungsmittelsicherheit in Bezug auf das Bestehen im Markt noch von vielen nicht erkannt wurde. 
2. Unter den internen Bedingungen der Wirtschaft wurde hervorgehoben: in den traditionell wirtschaftenden Betrieben wird der Umweltschutz wegen des chronischen Kapitalmangels hinter der Finanzierung der Funktionsfähigkeit, dem Ersatz für die Betriebsmittel in den Hintergrund verdrängt. Die ungelösten technologischen Komponenten der industriemäßigen Tierhaltung und die flächenmäßigen Grenzen der Stallmistdeponierung bereiten den Landwirten schwere Sorgen. Deshalb halten sie die umweltfreundliche Bewirtschaftung für kostenaufwendiger und die Risiken der umweltfreundlichen Produktion für größer. Sie schätzen die ertragsproduzierende Fähigkeit als gering, beziehungsweise als später realisierbar ein.

3. Unter den externen Bedingungen der Wirtschaft wurde erwähnt, dass die Ansprüche auf Ausschreibungen wegen Mangel an Staatshaushaltsmitteln nicht befriedigt werden können. Die praktische Wirkung der Umweltschutzmaßnahmen wird als minimal eingeschätzt. In Verbindung mit dem Nationalen Programm für Agrarumweltschutz haben sich zwei Personen geäußert: sie haben die Erfahrungen des ersten Jahres als günstig beurteilt und ihre Erwartung über positive künftige Veränderungen ausgedrückt.

\section{LITERATUR}

Belz, F. M. (2001): Integratives Öko-Marketing. Gabler Verlag, Deutscher Universitätsverlag, Wiesbaden

Csutora, M. (2001): Környezetgazdaságtan. CD anyag, Budapest (Umweltwirtschaftslehre, CD-Stoff)

Dyllick, T. (1989): Management der Umweltbeziehungen. Gabler Verlag, Wiesbaden

Dyllick, T.-Belz, F. (1996): Ökologie als Wettbewerbs-Faktor. In: Der Monat in Wirtschaft und Finanz, März, 16-19.

Dyllick, T.-Hamschmidt, J. (2000): Wirksamkeit und Leistung von Umweltmanagementsystemen. Hochschulverlag AG an der ETH Zürich

Kálmán, E. (2002): Környezetvédelmi együttműködés az ellátási láncok mentén. BKÁE, Környezettudományi Intézetének tanulmányai, 10. Budapest (Kooperation im Umweltschutz an den Versorgungsketten entlang. Studie)

Kósi, K. (1996): Környezeti menedzsment rendszerek a gyakorlatban I-II. Gazdálkodás, 4. 79-86.; 5. 77-81. (Umweltmanagement-Systeme in der Praxis I-II.)

Kovács, A. (1999): Környezeti marketing. In.: Marketing \& Management. 1. 47-51. (Umwelt-Marketing)
Meffert, H.-Kirchgeorg, M. (1998): Öko-Marketing Erfolgsvoraussetzungen und Entwicklungsperspektiven. In: Winter, G.: Das umweltbewusste Unternehmen, Verlag F. Vahlen, München, 785-803.

Meffert, H.-Ostmeier, H. (1990): Umweltschutz und Marketing. Berichte 8/90, Umweltbundesamt, Erich Schmidt Verlag, Berlin

Németh, P. (1999): Ökomarketing a 21. század küszöbén, 1.-2. rész. In.: Marketing \& Management 1. 41-46.; 2. 23-28. (Ökomarketing an der Schwelle des 21. Jahrhunderts)

Szabó, G.-Fésüs, I.-Balázs, K.-Katonáné Kovács, J. (2003): A Nemzeti Agrár-környezetvédelmi Program pályázatainak elemzése. In.: Gazdálkodás, 1. 26-39. (Analyse des Nationalen Programms für Agrarumweltschutz)

Szolnoki Gy-né Karkus, M. (1999): A zöld marketing és gazdasági környezete. Mezőgazda és Osiris Kiadó, Budapest, 207. (Das grüne Marketing und seine wirtschaftliche Umwelt)

Zsolnai, L. (1994): Gazdasági etika és ökológia. Aula, 2. 23-26. (Wirtschaftliche Ethik und Ökologie) 